\title{
Spatial comparison of areas at risk for schistosomiasis in the hilly and mountainous regions in the People's Republic of China: evaluation of the long-term effect of the 10-year World Bank Loan Project
}

\author{
Zhi-Jie Zhang ${ }^{1,2,3}$, Rong Zhu', N. Robert Bergquist ${ }^{5}$, Dong-Mei Chen 6 , Yue Chen 7 , Li-Juan \\ Zhang $^{4}$, Jia-Gang Guo ${ }^{4}$, Fei Zhao ${ }^{3}$, Qing-Wu Jiang ${ }^{1,2,3}$ \\ ${ }^{1}$ Department of Epidemiology, School of Public Health, Fudan University, Shanghai 200032, People's Republic \\ of China; ${ }^{2}$ Key Laboratory of Public Health Safety, Ministry of Education, Shanghai 200032, People's Republic \\ of China; ${ }^{3}$ Laboratory for Spatial Analysis and Modelling, School of Public Health, Fudan University, Shanghai \\ 200032, People's Republic of China; ${ }^{4}$ National Institute of Parasitic Diseases, Chinese Center for Disease \\ Control and Prevention, Shanghai 200025, People's Republic of China; Ingerod, Brastad, Sweden; ${ }^{6}$ Laboratory \\ of Geographic Information and Spatial Analysis, Department of Geography, Faculty of Arts and Science, \\ Queen's University, Kingston K7L 3N6, Ontario, Canada; ${ }^{7}$ Department of Epidemiology and Community \\ Medicine, Faculty of Medicine, University of Ottawa, Ottawa K1H 8M5, Ontario, Canada
}

\begin{abstract}
The long-term effectiveness of the mainly chemotherapy-based control strategy of the World Bank Loan Project (WBLP) for schistosomiasis control in Chinese hilly and mountainous regions was evaluated with a view to determine the best road forward. Based on the national database of schistosomiasis prevalence for the periods of 1999-2001 and 20072008 in the People's Republic of China, a Bayesian regression model was used for spatial comparison of schistosomiasis risk distribution between two periods taking account of all the potential risk factors simultaneously through two latent components of random effects: spatially correlated heterogeneities $(\mathrm{CH})$ and spatially uncorrelated heterogeneities (UH). Four different types of endemic areas were investigated: those that remained endemic despite control efforts (17 or $37.8 \%$ ), those that became non-endemic ( 9 or $20.0 \%$ ), those that reverted back to endemicity ( 7 or $15.6 \%$ ), and those with fluctuating endemicity (12 or $26.7 \%$ ). The overall prevalence of schistosomiasis was lower in 2007-2008 compared with that in 1999 2001, but the spatial distribution of risk remained similar. Compared to 1999-2001, the magnitude and range of risk even tended to be greater in 2007-2008. UH showed a fluctuating pattern, while CH increased gradually doubling over the two periods. There was no evidence for long-term effectiveness of the WBLP chemotherapy-based control strategy in this region. Controlling the effect of UH is still the main aspect of current schistosomiasis control strategy for the hilly and mountainous regions, but innovative methods are urgently needed for effectively controlling UH.
\end{abstract}

Keywords: schistosomiasis, strategy, spatial analysis, Bayesian statistics, People’s Republic of China.

Introduction

Schistosomiasis is a snail-transmitted trematode infection that ranks second only to malaria in terms of human suffering in the world (Steinmann et al., 2006; Zhang et al., 2009b; Zhou et al., 2010). Great progress has been made in controlling the oriental form, schistosomiasis japonica, the People's Republic of China(P. R. China), which instituted a successful,

\footnotetext{
Corresponding author:

Zhi-Jie Zhang

Department of Epidemiology, School of Public Health

Fudan University, Shanghai 200032, People's Republic of China

Tel./Fax +862154237410

E-mail: zhj_zhang@fudan.eud.cn
}

national control programme more than 60 years ago (McManus et al., 2010; Utzinger et al., 2010; Gray et al., 2011). The number of infected people was reduced from 11.6 million in the mid-1950s to less than one million in 2003, and for infected cattle the corresponding numbers were 1.2 million and less than one million, respectively (Zhou et al., 2005a, 2007). Simultaneously, the area infested by the intermediate host snail, Oncomelania hupensis, decreased from $14,300 \mathrm{~km}^{2}$ in the mid-1950s to $3,787 \mathrm{~km}^{2}$ in 2003 , but the infested areas have rebounded compared to the lowest level in 2001 (3,436 km²) (Chen et al., 2002; Zhou et al., 2005a, 2007).

According to the geography of the endemic areas and the ecological characteristics of the snail, the endemic regions in P. R. China have been stratified into three 
types: plain regions with waterway networks, lake and marshland regions, and hilly and mountainous regions (Zhang et al., 2008; Yang et al., 2009; Zhou et al., 2010). Current endemic regions have been largely confined to the lake and marshland regions in the provinces of Hunan, Hubei, Anhui, Jiangxi and Jiangsu, and the hilly and mountainous regions in the provinces of Yunnan and Sichuan (Utzinger et al., 2005; Zhao et al., 2005; Zhou et al., 2005a). Transmission of schistosomiasis in the plain regions with waterway networks, was interrupted long ago through the snail elimination (Zhang et al., 2009a; Zhou et al., 2010). According to the latest report of the national schistosomiasis control programme, only an estimated $1.3 \%$ of the total area remained snail-ridden with $2 \%$ of the population infected in Yunnan and Sichuan in 2009 (Hao et al., 2010). These low figures give hope that the hilly and mountainous regions may become the next areas where schistosomiasis transmission can be successfully interrupted.

P. R. China has set an ambitious goal for schistosomiasis control. Transmission in the hilly and mountainous regions is expected to be interupted by 2015 (Engels et al., 2005; Utzinger et al., 2005; Zhou et al., 2005a). The current strategy implemented in these areas remains that developed from the WBLP mainly chemotherapy-based integrated control from the 1990s. Previous assessment reports concluded that the WBLP successfully reduced the morbidity of schistosomiasis in humans and livestock in those areas (Zhang and Wong, 2003; Chen et al., 2005), but they were based on short-term control efforts without considering the spatial aspects, which raises many queries. What is the long-term control effect of WBLP on schistosomiasis? What results can we obtain if an evaluation was to be conducted from the spatial point of view? To achieve the ultimate goals of transmission interruption and with the aim of sustainable control in mind, should we switch the morbidity control strategy to a transmission control strategy in these areas? The situation remains poorly understood and the questions are vital for developing an effective strategy for controlling the disease.

This study aims at assessment of the long-term effectiveness of the WBLP strategy for schistosomiasis control from the spatial point of view based on data from the hilly and mountainous regions for the periods of 1999-2001 and 2007-2008. A Bayesian-based regression model was adopted to compare these two periods for the spatial distribution of risk with schistosomiasis-related risk factors adjusted through two latent components of random effects: spatially correlated heterogeneity $(\mathrm{CH})$ and uncorrelated heterogeneity (UH). The former component is an indicator of all schistosomiasis-related risk factors with spatial autocorrelations, e.g. land surface temperature, while the latter represents the integrated effects of all risk factors, which are independent of space such as frequency of water contact and the like.

\section{Materials and methods}

\section{Parasitological data}

The WBLP started in Sichuan and Yunnan provinces in 1992, ceased in Sichuan by the end of 1998 but continued until the end of 2001 in Yunnan. However, the control activities in Sichuan, following the operational plan of the WBLP, continued with funding from the provincial government (Chen et al., 2005). Thus, both provinces were subject to the same control activities until the end of 2001.

The national schistosomiasis datasets were maintained and managed by the Fudan University (formerly Shanghai Medical University) before 2004, while this work has been taken over by the National Institute of Parasitic Diseases in Shanghai (formerly the Institute of Parasitic Diseases), Chinese Center for Disease Control and Prevention (China CDC) since 2005. The parasitological databases for the hilly and mountainous regions in Sichuan and Yunnan, used in this study, were kindly provided by the Fudan University and China CDC. The datasets are countybased, including information on reported schistosomiasis cases and population at risk in each county, and cover two periods: 1999-2001 and 2007-2008.

\section{Base map}

The county-based, digitized polygon maps of Sichuan and Yunnan provinces at the scale of 1:250,000 were extracted from an existing national map. It was used to link the attributed data to establish a spatial database for schistosomiasis (Zhang et al., 2008, 2009a, b). During the 10-year study period, the administrative divisions changed slightly. To keep the spatial structure of schistosomiasis databases consistent during the study period, the administrative divisions in 2008 were chosen as the standard spatial structure, while all the datasets for all other years were modified accordingly, including merging and splitting of polygons. Merging was a straightforward move resulting in adding schistosomiasis data together, while splitting required re-allocating the data based on 
the spatial proportions of the new polygons versus the original ones.

\section{Statistical analysis}

Firstly, descriptive statistics such as median and $95 \%$ confidence interval (CI) were calculated to describe the basic characteristics of the schistosomiasis data. Secondly, the study region was classified into one non-endemic region where no cases had been reported in any of the periods and four types of endemic regions as follows:

(i) constantly endemic areas with cases reported every year covered by the study;

(ii) new or re-emerged areas with no cases reported in 1999-2001 but in 2007-2008;

(iii) new non-endemic areas with cases reported in 1999-2001, but not in 2007-2008; and

(iv) fluctuating areas with cases reported once or twice in the 1999-2001 period and once either in 2007 or in 2008.

The spatial distribution of these four endemic types of schistosomiasis areas was mapped out and an overlay produced to fit the base map, i.e. the digitized polygon map of the hilly and mountainous regions. Thirdly, a Bayesian regression model was used to analyse the area-based relative risk (RR) by treating the schistosomiasis-related risk factors sorted as two latent components of random effects. These factors were (i) $\mathrm{CH}$, an indicator of all spatially autocorrelated risk factors modeled using the conditional autoregressive structure (AR) (Besag et al., 1991) and (ii) UH, the integrated effects of non-spatial risk factors such as the frequency of water contact (with water harbouring infected snails). The relative magnitude of $\mathrm{CH}$ and $\mathrm{UH}$ were analysed with the aim of gaining new insights for future control strategies. By including all possible risk factors, the predicted risk becomes increasingly precise. The idea of this model was first introduced by Clayton and Kaldor (1987) and further developed by Besag et al. (1991). Following the example of Lawson et al. (2003) the risk can be estimated as follows:

$$
\begin{gathered}
O_{i} \sim \text { Poisson }\left(E_{i} \Theta_{i}\right) \\
\log \Theta_{i}=\alpha+c_{i}+u_{i}
\end{gathered}
$$

where $O_{i}$ and $E_{i}$ represent observed and expected schistosomiasis cases, respectively; $\Theta_{i}$ is the area-based $\mathrm{RR}$ and the baseline risk assuming the effects of $c_{i}$ $(\mathrm{CH})$ and $u_{i}(\mathrm{UH})=0$. The latter two $\left(c_{i}\right.$ and $\left.u_{i}\right)$ are modeled using the following formulas:

$$
\left[c_{i} \mid c_{j}, i \neq j, \tau_{c}^{2}\right] \sim \mathrm{N}\left(\frac{\sum_{j} c_{j} w_{i j}}{\sum_{j} w_{i j}}, \frac{\tau_{c}^{2}}{\sum_{j} w_{i j}}\right)
$$

$$
u_{i} \sim N\left(0, \tau_{u}^{2}\right)
$$

where $w_{i j}$ is the weight of the neighbour $j$ for area $i, w_{i j}$ $=1$ if $i$ and $j$ are neighbours, otherwise $w_{i j}=0 ; \tau_{c}^{2}$ and $\tau_{u}^{2}$ are the variance of $c_{i}$ and $u_{i}$, respectively.

The Bayesian technique was applied to fit the model. Parameters $\tau_{c}^{2}$ and $\tau_{u}^{2}$ control the variability of $\mathrm{CH}$ and $\mathrm{UH}$ effects, for which prior distributions were specified using the same vague gamma prior distributionsGamma $(0.5,0.0005)$. For the baseline risk $\alpha$, the vague normal prior distribution- $N(0,0.0001)$ was used for its prior distribution. Two-chain Markov chain Monte Carlo (MCMC) simulations with 30,000 iterations were run. The first 10,000 iterations were discarded as burn-in and the remaining 20,000 iterations of both chains were used to summarise and infer the estimated parameters. The model convergence was judged by a visual inspection of the time-series plots of each parameter and by computing the Gelman-Rubin statistics (Gelman and Rubin, 1992). Finally, the changes of the baseline risk $\alpha$, the variation of $\mathrm{UH}$ and $\mathrm{CH}$, the area-based RR, posterior predicted probability of $\mathrm{RR}>1$, the ratio of $\mathrm{UH}$ to $\mathrm{CH}$ and model residuals were compared and analysed.

\section{Results}

The total number of endemic counties with schistosomiasis cases fell slightly. The prevalence of schistosomiasis in the period of 2007-2008 was lower than that in the period of 1999-2001, but in each period there were some signs of rebounding. However, the level $(95 \% \mathrm{CI})$ of schistosomiasis prevalence was continuously reduced (Table 1). Fig. 1 shows that the different types of areas endemic for schistosomiasis were co-mingled in the middle part of the hilly and mountainous regions with the following distribution: constant endemic areas (17 or $37.8 \%$ ), new non-endemic areas $(9$ or $20.0 \%$ ), new or re-emerged areas (7 or $15.6 \%$ ) and fluctuating endemic areas (12 or $26.7 \%$ ). The fluctuating regions were all in Sichuan province, but the new or re-emerged areas appeared in both Sichuan and Yunnan.

The baseline risk of schistosomiasis tended to decrease gradually, except for a large rebound in 2001, the last year of WBLP activities. The variation of $\mathrm{CH}$ increased gradually and was twice as high in $2007 / 2008$ compared to 1999 . However, the variation 
Table 1. Summary of the prevalence of S. japonicum in the hilly and mountainous regions of Sichuan and Yunnan province, P. R. China.

\begin{tabular}{ccccccrr}
\hline Year & $\mathrm{N}$ & $\begin{array}{c}\text { Min } \\
\left(10^{-5}\right)\end{array}$ & $\begin{array}{c}\text { Q1 } \\
\left(10^{-3}\right)\end{array}$ & $\begin{array}{c}\text { Median } \\
(10-2)\end{array}$ & $\begin{array}{c}\text { Q3 } \\
\left(10^{-2}\right)\end{array}$ & $\begin{array}{c}\text { Max } \\
\left(10^{-1}\right)\end{array}$ & $\begin{array}{c}P 2.5 \\
\left(10^{-5}\right)\end{array}$ \\
\hline 1999 & 37 & 5.31 & 1.36 & 1.49 & 1.85 & 1.17 & 5.31 \\
2000 & 28 & 0.91 & 0.12 & 0.24 & 1.22 & 0.67 & 0.91 \\
2001 & 34 & 0.59 & 0.21 & 0.33 & 0.89 & 0.60 & 0.59 \\
2007 & 34 & 0.87 & 0.20 & 0.05 & 0.12 & 0.07 & 0.87 \\
2008 & 25 & 14.39 & 0.60 & 0.14 & 0.27 & 0.03 & 14.39 \\
\hline
\end{tabular}

of UH showed a fluctuating pattern and was strongly dominated $\mathrm{CH}$ at all times (Table 2). Fig. 2 shows that areas at risk for schistosomiasis $(\mathrm{RR}>1)$ were relatively stable spatially between the time periods studied. In Yunnan, the range and size of risk areas increased in the period of 1999-2001 but decreased in 2007-2008, while the areas at risk for schistosomiasis rebounded spatially to a certain degree with a more dispersed distribution pattern in Sichuan. As can be seen in Fig. 3, the posterior probabilities were also higher for the regions where the risk for schistosomiasis was higher $(\mathrm{RR}>1)$. The $\mathrm{UH} / \mathrm{CH}$ ratio was relatively large $(>1)$ over the entire time span studied in the areas with reported schistosomiasis cases with the magnitude and range even expanding in 2007-2008 (Fig. 4). Residuals of the Bayesian model were all between -1 and 1 . As no outliers were found, the result is not displayed here (data available upon request).

\section{Discussion}

Previous studies have documented distinct epidemiological and ecological features of schistosomiasis in the hilly and mountainous regions which are not present in other areas such as lake and marshland and plain regions with waterway networks. For example, the snail habitats in the hilly and mountainous regions are typically small and usually scattered over the terrain in separate patches mainly distributed along ditches, irrigation canals and river systems. The situation with widely distributed, small Schistosoma japonicum transmission foci, rather than continuous endemic areas, renders schistosomiasis control in the hilly and mountainous regions more difficult to deal with (Seto et al., 2002; Xu et al., 2004; Gurarie and King, 2005; Liang et al., 2006).

The implementation of the WBLP in 1992 provided

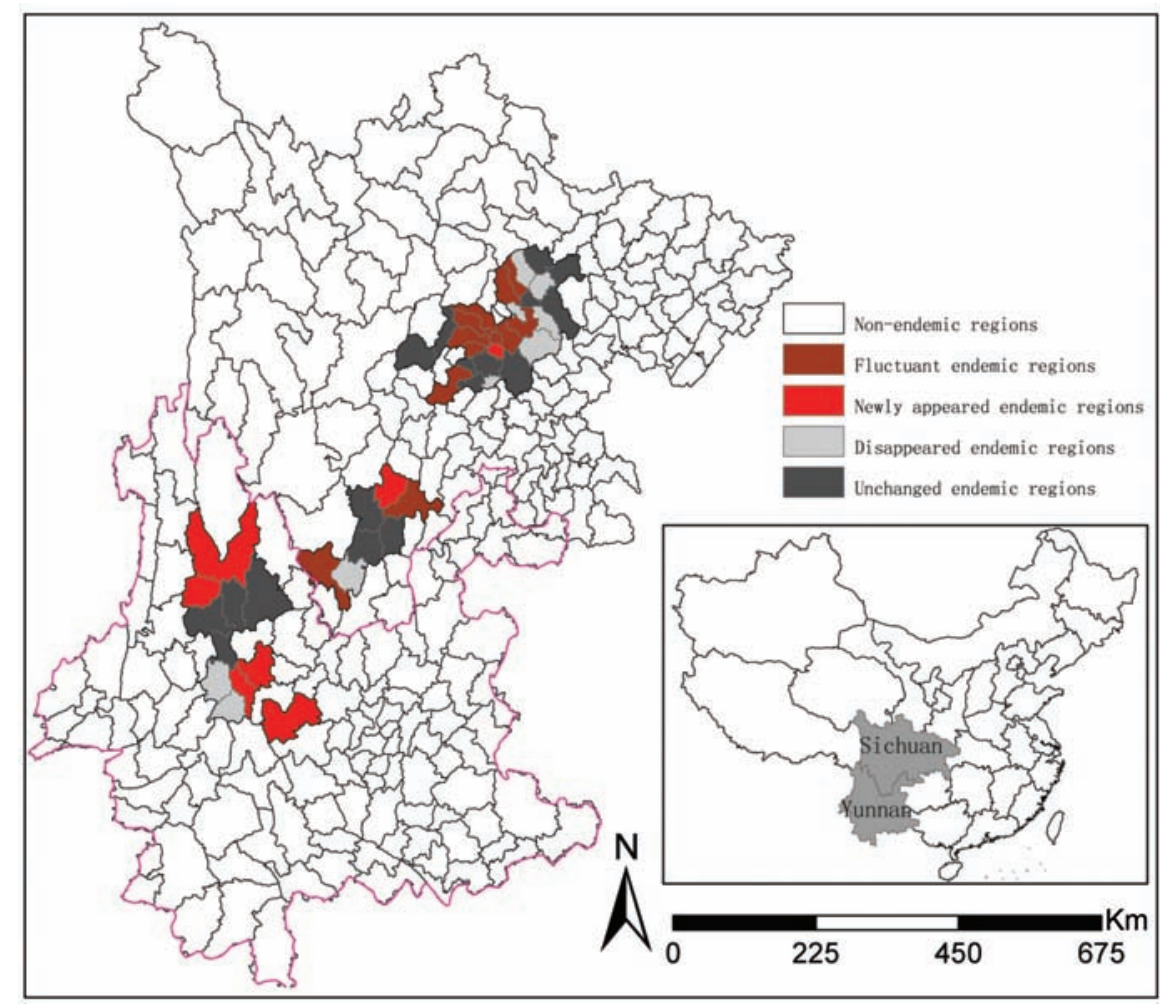

Fig. 1. Distribution of the different types of areas endemic for schistosomiasis. 
Table 2. Parameter estimates of the Bayesian model.

\begin{tabular}{|c|c|c|c|c|c|c|}
\hline Year & Variable & Median & SD & MC error & $P 2.5$ & P 97.5 \\
\hline \multirow{3}{*}{1999} & Overall risk & 0.25 & 0.09 & 0.01 & 0.12 & 0.44 \\
\hline & $\mathrm{SD}(\mathrm{CH})$ & 0.03 & 0.03 & 0.00 & 0.01 & 0.13 \\
\hline & $\mathrm{SD}(\mathrm{UH})$ & 6.11 & 0.88 & 0.02 & 4.69 & 8.10 \\
\hline \multirow{3}{*}{2000} & Overall risk & 0.16 & 0.07 & 0.00 & 0.05 & 0.28 \\
\hline & $\mathrm{SD}(\mathrm{CH})$ & 0.04 & 0.20 & 0.01 & 0.01 & 0.73 \\
\hline & $\mathrm{SD}(\mathrm{UH})$ & 8.31 & 1.24 & 0.04 & 6.14 & 10.90 \\
\hline \multirow{3}{*}{2001} & Overall risk & 0.49 & 0.30 & 0.02 & 0.10 & 1.05 \\
\hline & $\mathrm{SD}(\mathrm{CH})$ & 0.04 & 0.05 & 0.00 & 0.01 & 0.19 \\
\hline & $\mathrm{SD}(\mathrm{UH})$ & 7.85 & 1.20 & 0.04 & 5.71 & 10.30 \\
\hline \multirow{3}{*}{2007} & Overall risk & 0.03 & 0.02 & 0.00 & 0.01 & 0.08 \\
\hline & $\mathrm{SD}(\mathrm{CH})$ & 0.06 & 0.22 & 0.01 & 0.02 & 0.81 \\
\hline & $\mathrm{SD}(\mathrm{UH})$ & 3.62 & 0.54 & 0.02 & 2.78 & 4.87 \\
\hline \multirow{3}{*}{2008} & Overall risk & 0.01 & 0.01 & 0.00 & 0.00 & 0.04 \\
\hline & $\mathrm{SD}(\mathrm{CH})$ & 0.06 & 0.12 & 0.01 & 0.02 & 0.49 \\
\hline & $\mathrm{SD}(\mathrm{UH})$ & 5.52 & 1.03 & 0.04 & 4.06 & 8.08 \\
\hline
\end{tabular}

Note: $\mathrm{SD}=$ standard error; $\mathrm{UH}=$ uncorrelated heterogeneity; $\mathrm{CH}=$ correlated heterogeneity; $\mathrm{MC}$ error =Monte Carlo error.
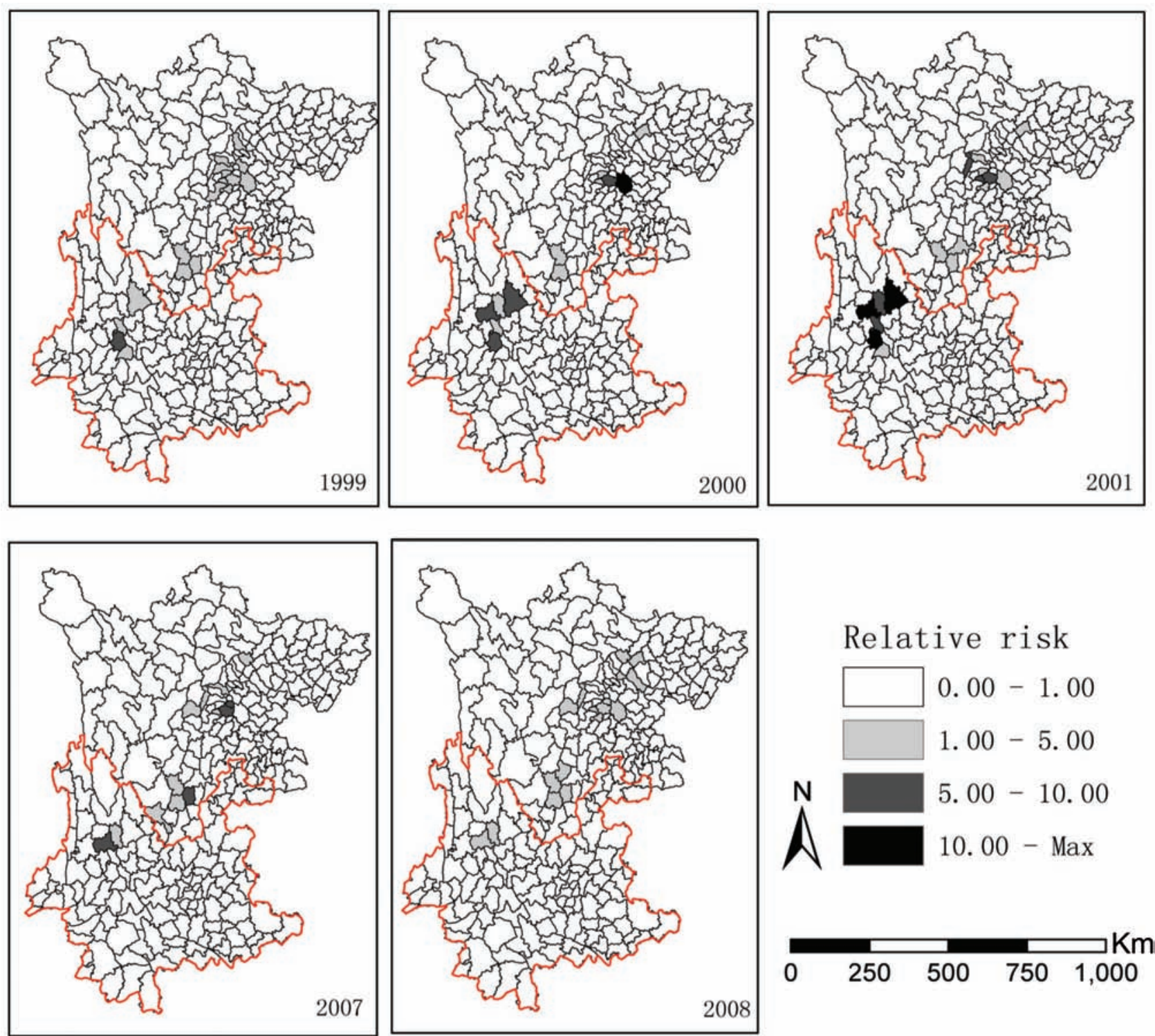

Relative risk

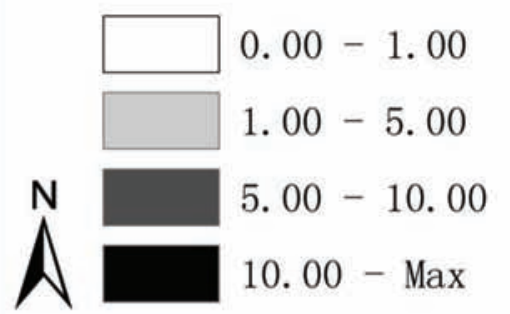
$0 \quad 250 \quad 500 \quad 750 \quad 1,000$

Fig. 2. Predicted relative risk distribution according to the Bayesian model. 

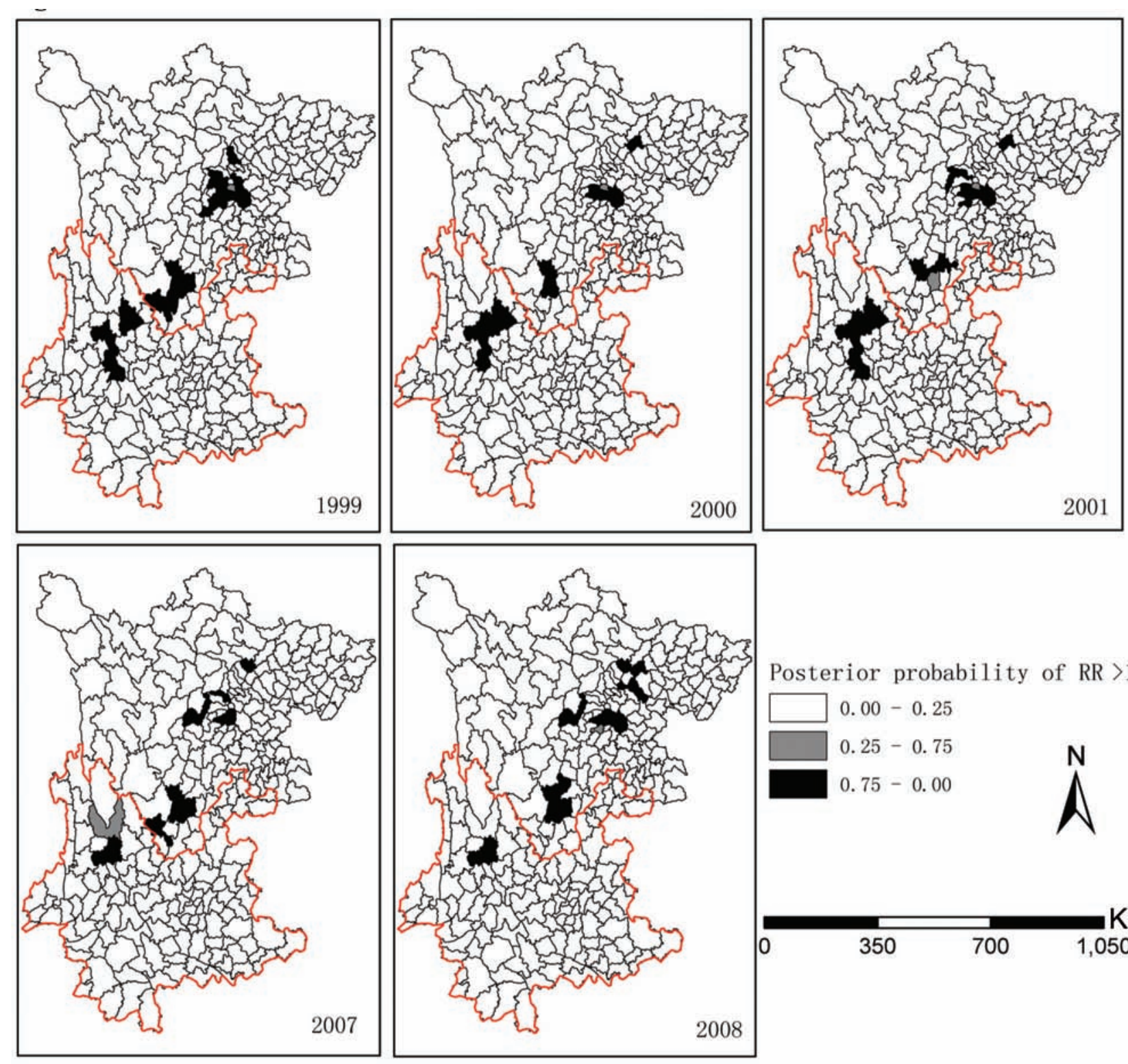

Posterior probability of RR $>1$
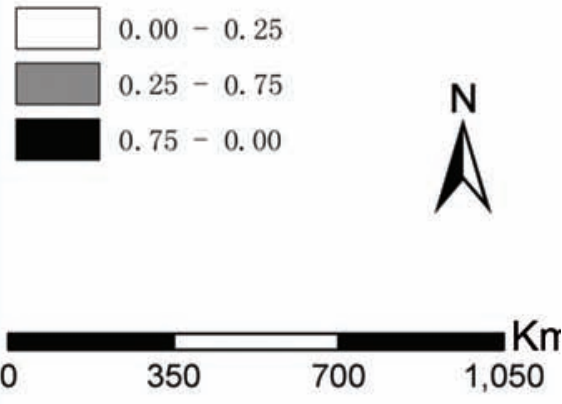

Fig. 3. Distribution of posterior probability of $\mathrm{RR}>1$ according to the Bayesian model.

a new impetus for schistosomiasis control resulting in a spectacular reduction of both prevalence and intensity of infection, both in humans, cattle and water buffaloes (Ross et al., 1997; Zhang and Wong, 2003; Chen et al., 2005). After the programme was terminated however, there was a shortfall of financial resources required to maintain the achievements made, let alone making further progress (Chen et al., 2005; Zhao et al., 2005; Liang et al., 2006). Researchers soon discovered rebounding schistosomiasis in the hilly and mountainous regions, including a re-emergence in areas where transmission had previously been interrupted (Engels et al., 2005; Liang et al., 2006; Yang et al., 2009). Hence, the sustainability the WBLP model of schistosomiasis control was repeatedly questioned (Utzinger et al., 2003, 2010; Zhang et al., 2009b; Seto et al., 2011; Zhou et al., 2011). To date, two studies have discussed the shortterm impact of the WBLP on schistosomiasis prevalence, intensity and control (Zhang and Wong, 2003; Chen et al., 2005), but we have not seen any studies concerning the long-term implications of the WBLP. To our knowledge, this is the first study evaluating the long-term control effect of WBLP in the hilly and mountainous regions.

To accurately predict the risk of schistosomiasis, all potential risk factors needed to be included in the model. However, putting all risk factors in one model is impractical because either the necessary datasets are not available or the disease mechanisms are not com- 

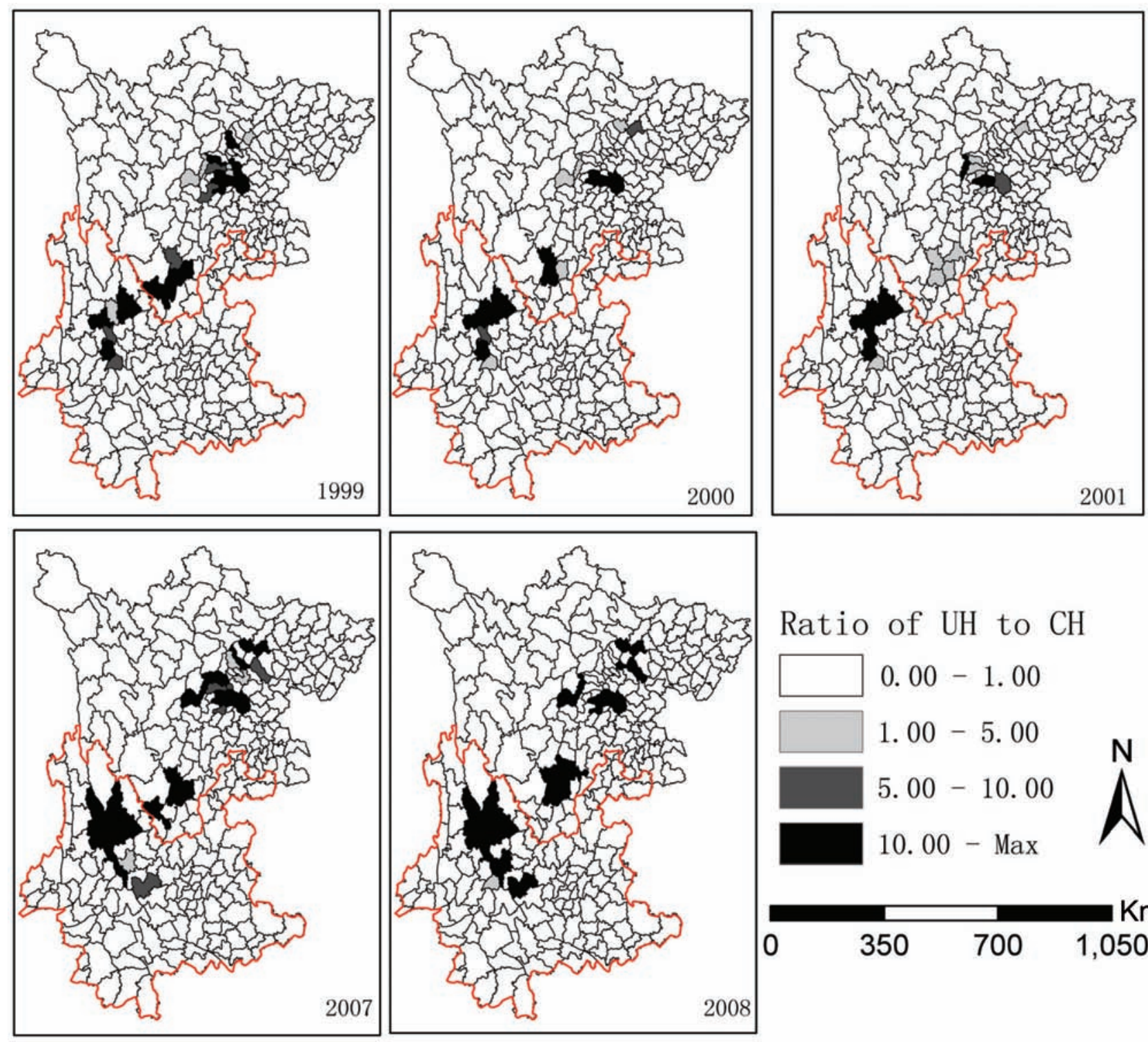

\section{Ratio of $\mathrm{UH}$ to $\mathrm{CH}$}

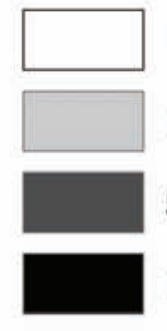

$0.00-1.00$

$1.00-5.00$

$5.00-10.00$

10. 00 - Max

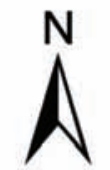

$0 \quad 350 \quad 700 \quad 1,050$

Fig. 4. Spatial distribution of the uncorrelated heterogeneity $(\mathrm{UH})$ to the correlated heterogeneity $(\mathrm{CH})$ ratio.

pletely clear (Malone et al., 2010; Magalhães et al., 2011; Spear, 2011). To avoid this, we used a Bayesian model in with representation of the random effects of all the possible risk factors related to schistosomiasis sorted into two groups, one consisting of spatially correlated heterogeneity and one concerning spatially uncorrelated heterogeneity. The snail is the most controllable factor, which is in correspondence to the traditional transmission control strategy of schistosomiasis in P. R. China. UH is an indicator of the combined effect of human behavioural factors such as frequency of water contact, personal protection, health education, etc. all have a bearing on the role of the snail. The chemotherapy-based morbidity control strategy is a parallel control approach (Clayton and Kaldor,
1987; Besag et al., 1991; Lawson et al., 2003). Thus, by analysing the relative magnitude of $\mathrm{CH}$ and $\mathrm{UH}$, we were able to obtain some insight that could prove important in the planning of an improved strategy to control schistosomiasis. Also, by taking $\mathrm{CH}$ and $\mathrm{UH}$ into consideration as means of adjusting the unmeasured risk factors of schistosomiasis, the predicted schistosomiasis risk becomes more precise.

It was found that the prevalence of schistosomiasis was confined to a narrower range between the two study periods, and only small variations were observed. The overall endemic level in the hilly and mountainous regions was reduced in the period of 2007-2008. The main strategy of the WBLP was to control morbidity due to schistosomiasis, which is 
reflected by the changes of $\mathrm{UH}$ investigated in this study. Continuous drug treatment in the schistosomiasis risk regions should theoretically promote a trend towards homogenous $\mathrm{UH}$ values, i.e. the variability should continue to decline. Compared to 1999-2001, UH did indeed fall in 2007-2008, but fluctuated within each period and rebounding strongly in 2008 almost reaching the level of 1999 . This suggests that the long-term effect of the chemotherapy-based control strategy may not be stable. There may be multiple reasons for this, including decreased funding, lack of coordination between the health, agriculture and water resource sectors, increased cattle/human mobility through trading/traveling, construction of additional irrigation projects, among others (Wang et al., 2004; Liang et al., 2006; Yang et al., 2009).

The value of $\mathrm{CH}$ tended to continuously increase. There is a possibility that the effect from the intermediate host snail continues increasing all the time although its absolute size was largely smaller than $\mathrm{UH}$ (Liang et al., 2006). This reflects well the limitation of the WBLP control strategy, which treated snail control only as a complementary strategy. Besides, the rebound of the schistosomiasis epidemic in each period, the re-emerging or new endemic areas and the considerable fluctuation in the schistosomiasis regions in the second period of 2007-2008 also suggest that the effect of WBLP control strategy cannot be sustained for the long term.

The areas at risk for schistosomiasis predicted by aBayesian model were spatially relatively stable and continue to be so. Although the WBLP strategy in the endemic areas in the hilly and mountainous regions resulted in a reduced magnitude of the prevalence of schistosomiasis, it failed to effectively reduce the extent of the endemic areas. Based on previous experience (Chen et al., 2005; Zhao et al., 2005; Zhang et al., 2009b; Zhou et al., 2010, 2011), transmission must also be curtailed and the only way to achieve that is to increase the emphasis on snail control. From the spatial distribution of the $\mathrm{UH} / \mathrm{CH}$ ratio, it is clear that UH dominated current control activities in the schistosomiasis risk regions. Thus, the progress achieved underlines the need to reduce $\mathrm{UH}$ in the hilly and mountainous regions. Moreover, the areas at risk for schistosomiasis dominated by UH expanded, suggesting that the effectiveness of chemotherapy-based control strategy from WBLP had declined. At the current stage, it is most important to reduce the effect caused by UH in order to progress in controlling schistosomiasis at the same time as new innovative control strategis are contemplated. For example, Wang et al. (2009a, b) proposed a newly integrated control strategy aiming at reducing the roles of humans, cattle and water buffaloes as snail infection sources. This may be an alternative way to further control the transmission of schistosomiasis. However, there is a concern that it cannot interrupt the schistosome life cycle because there are more than 40 different, definitive hosts that can become infected by S. japonicum (Zhou et al., 2005b; Wang et al., 2006). Clearly, it would be possible to stop schistosomiasis transmission by eliminating its sole intermediate host. However, there is evidence that the schistosome life cycle can be effectively interupted already by reducing the number of snails, e.g. the threshold value for the transmission (Tanaka and Tsuji, 1997; Chen et al., 2005).

\section{Conclusion}

The long-term control effect of the WBLP's schistosomiasis control strategy in the hilly and mountainous regions is unstable, underlining the need for developing an improved strategy. Controlling UH remains the main aspect of schistosomiasis control strategy for the consolidation of the achievements made previously in these areas. Since the chemotherapy-based, integrated control strategy has been shown to be unable to break the transmission cycle, novel approaches are urgently needed. Innovative snail control measures are also needed to reduce the $\mathrm{CH}$ effect and prevent it from becoming a major factor in areas at risk for schistosomiasis in future.

\section{Acknowledgements}

This work was financially supported by the National Natural Science Foundation of China (grant nos. 81102167 and 81172609), Specialized Research Fund for the Doctoral Program of Higher Education, SRFDP (grant no. 20110071120040) and A Foundation for the Author of National Excellent Doctoral Dissertation of PR China (FANEDD) (grant number 201186). The funding agencies had no role in study design, data collection and analysis, decision to publish, or preparation of the manuscript.

\section{References}

Besag J, York J, Mollie J, 1991. Bayesian image restoration, with two applications in spatial statistics. Ann Inst Statist Math 43, 1-20.

Chen XY, Jiang QW, Wang LY, Zhao GM, Zhao Q, Gu GA, Wei JG, Hao Y, 2002. Schistosomiasis situation in the People's Republic of China in 2001. Chin J Schisto Contr 14, 241-243. 
Chen XY, Wang LY, Cai JM, Zhou XN, Zheng J, Guo JG, Wu $\mathrm{XH}$, Engels D, Chen MG, 2005. Schistosomiasis control in China: the impact of a 10-year World Bank Loan Project (1992-2001). Bull World Health Organ 83, 43-48.

Clayton D, Kaldor J, 1987. Empirical Bayes estimates of agestandardized relative risks for use in disease mapping. Biometrics 43, 671-681.

Engels D, Wang LY, Palmer KL, 2005. Control of schistosomiasis in China. Acta Trop 96, 67-68.

Gelman A, Rubin DB, 1992. Inference from iterative simulations using multiple sequences. Stat Sci 7, 7457-7472.

Gray DJ, Ross AG, Li YS, McManus DP, 2011. Diagnosis and management of schistosomiasis. BMJ 342, d2651.

Gurarie D, King CH, 2005. Heterogeneous model of schistosomiasis transmission and long-term control: the combined influence of spatial variation and age-dependent factors on optimal allocation of drug therapy. Parasitology 130, 49-65.

Hao Y, Zheng H, Zhu R, Guo JG, Wang LY, Chen Z, Zhou XN, 2010. Schistosomiasis situation in People's Republic of China in 2009. Chin J Schisto Contr 22, 521-527.

Lawson AB, Browne WJ, Vidal Rodeiro CL, 2003. Disease mapping with WinBUGS and MLwiN. John Wiley \& Sons Ltd, UK, pp. 123-128.

Liang S, Yang C, Zhong B, Qiu D, 2006. Re-emerging schistosomiasis in hilly and mountainous areas of Sichuan, China. Bull World Health Organ 84,139-144.

Magalhães RJ, Clements ACA, Patil AP, Gething PW, Brooker S, 2011. The applications of model-based geostatistics in helminth epidemiology and control. Adv Parasitol 74, 267296.

Malone JB, Yang GJ, Leonardo L, Zhou XN, 2010. Implementing a geospatial health data infrastructure for control of Asian schistosomiasis in the People's Republic of China and the Philippines. Adv Parasitol 73, 71-100.

McManus DP, Gray DJ, Li Y, Feng Z, Williams GM, Stewart D, 2010. Schistosomiasis in the People's Republic of China: the era of the Three Gorges Dam. Clin Microbiol Rev 23, 442466.

Ross AGP, Li YS, Sleigh AC, McManus DP, 1997. Schistosomiasis control in the People's Republic of China. Parasitol Today 13, 152-155.

Seto E, Xu B, Liang S, Gong P, Wu WP, George D, Qiu DC, Gu XG, Spear R, 2002. The use of remote sensing for predictive modeling of schistosomiasis in China. Photogramm Eng Rem Sens 68,167-174.

Seto EYW, Wong BK, Lu D, Zhong B, 2011. Human schistosomiasis resistance to praziquantel in China: should we be worried? Am J Trop Med Hyg 85, 74-82.

Spear RC, 2011. Internal versus external determinants of Schistosoma japonicum transmission in irrigated agricultural villages. J R Soc Interface 9, 272-282.

Steinmann P, Keiser J, Bos R, Tanner M, Utzinger J, 2006.
Schistosomiasis and water resources development: systematic review, meta-analysis, and estimates of people at risk. Lancet Infect Dis 6, 411-425.

Tanaka H, Tsuji M, 1997. From discovery to eradication of schistosomiasis in Japan: 1847-1996. Int J Parasitol 27, 1465 1480 .

Utzinger J, Bergquist R, Olveda R, Zhou XN, 2010. Important helminth infections in Southeast Asia: diversity, potential for control and prospects for elimination. Adv Parasitol 72, 1-30.

Utzinger J, Bergquist R, Xiao SH, Singer BH, Tanner M, 2003. Sustainable schistosomiasis control-the way forward. Lancet 362, 1932-1934.

Utzinger J, Zhou XN, Chen MG, Bergquist R, 2005. Conquering schistosomiasis in China: the long march. Acta Trop 96, 69-96.

Wang LD, Chen HG, Guo JG, Zeng XJ, Hong XL, Xiong JJ, Wu XH, Wang XH, Wang LY, Xia G, Hao Y, Chin DP, Zhou XN, 2009a. A strategy to control transmission of Schistosoma japonicum in China. N Engl J Med 360, 121-128.

Wang LD, Guo JG, Wu XH, Chen HG, Wang TP, Zhu SP, Zhang ZH, Steinmann P, Yang GJ, Wang SP, Wu ZD, Wang LY, Hao Y, Bergquist R, Utzinger J, Zhou XN, 2009b. China's new strategy to block Schistosoma japonicum transmission: experiences and impact beyond schistosomiasis. Trop Med Int Health 14, 1475-1483.

Wang RB, Wang TP, Wang LY, Guo JG, Yu Q, Xu J, Gao FH, Yin ZC, Zhou XN, 2004. Study on the re-emerging situation of schistosomiasis epidemics in areas already under control and interruption. Zhonghua Liu Xing Bing Xue Za Zhi 25, 564-567.

Wang TP, Shrivastava J, Johansen MV, Zhang SQ, Wang FF, Webster JP, 2006. Does multiple hosts mean multiple parasites? Population genetic structure of Schistosoma japonicum between definitive host species. Int J Parasitol 36, 1317-1325.

Xu B, Gong P, Biging G, Liang S, Seto E, Spear R, 2004. Snail density prediction for schistosomiasis control using IKONOS and ASTER images. Photogramm Eng Rem Sens 70, 12851294.

Yang K, Zhou XN, Wu XH, Steinmann P, Wang XH, Yang GJ, Utzinger J, Li HJ, 2009. Landscape pattern analysis and Bayesian modeling for predicting Oncomelania hupensis distribution in Eryuan county, People's Republic of China. Am J Trop Med Hyg 81, 416-423.

Zhang W, Wong CM, 2003. Evaluation of the 1992-1999 World Bank Schistosomiasis Control Project in China. Acta Trop 85, 303-313.

Zhang Z, Carpenter TE, Chen Y, Clark AB, Lynn HS, Peng W, Zhou Y, Zhao G, Jiang Q, 2008. Identifying high-risk regions for schistosomiasis in Guichi, China: a spatial analysis. Acta Trop 107, 217-223.

Zhang Z, Clark AB, Bivand R, Chen Y, Carpenter TE, Peng W, Zhou Y, Zhao G, Jiang Q, 2009a. Nonparametric spatial 
analysis to detect high-risk regions for schistosomiasis in Guichi, China. Trans R Soc Trop Med Hyg 103,1045-1052.

Zhang ZJ, Carpenter TE, Lynn HS, Chen Y, Bivand R, Clark AB, Hui FM, Peng WX, Zhou YB, Zhao GM, Jiang QW, 2009b. Location of active transmission sites of Schistosoma japonicum in lake and marshland regions in China. Parasitology 136, 737-746.

Zhao GM, Zhao Q, Jiang QW, Chen XY, Wang LY, Yuan HC, 2005. Surveillance for schistosomiasis japonica in China from 2000 to 2003. Acta Trop 96, 288-295.

Zhou XN, Bergquist R, Leonardo L, Yang GJ, Yang K, Sudomo M, Olveda R, 2010. Schistosomiasis japonica control and research needs. Adv Parasitol 72, 145-178.

Zhou XN, Guo JG, Wu XH, Jiang QW, Zheng J, Dang H, Wang XH, Xu J, Zhu HQ, Wu GL, Li YS, Xu XJ, Chen HG, Wang TP, Zhu YC, Qiu DC, Dong XQ, Zhao GM, Zhang SJ, Zhao
NQ, Xia G, Wang LY, Zhang SQ, Lin DD, Chen MG, Hao Y, 2007. Epidemiology of schistosomiasis in the People's Republic of China, 2004. Emerg Infect Dis 13, 1470-1476.

Zhou XN, Wang LY, Chen MG, Wu XH, Jiang QW, Chen XY, Zheng J, Utzinger J, 2005a. The public health significance and control of schistosomiasis in China - then and now. Acta Trop 96, 97-105.

Zhou XN, Jiang QW, Sun LP, Wang TP, Hong QB, Zhao GM, Wen LY, Yin ZC, Wu XH, Lin DD, 2005b. Schistosomiasis control and surveillance in China. Chin J Schisto Cont 17, 161-165.

Zhou YB, Liang S, Chen GX, Rea C, He ZG, Zhang ZJ, Wei JG, Zhao GM, Jiang QW, 2011. An integrated strategy for transmission control of Schistosoma japonicum in a marshland area of China: findings from a five-year longitudinal survey and mathematical modeling. Am J Trop Med Hyg 85, 83-88. 